\title{
Corela
}

Cognition, représentation, langage

HS-22 | 2017

Prédication et prépositions en anglais

\section{Grammaire cognitive des prépositions : épistémologie et applications}

Jean-Rémi Lapaire

\section{OpenEdition}

Journals

Édition électronique

URL : http://journals.openedition.org/corela/5003

DOI : $10.4000 /$ corela.5003

ISSN : $1638-573 \mathrm{X}$

Éditeur

Cercle linguistique du Centre et de l'Ouest - CerLICO

Référence électronique

Jean-Rémi Lapaire, « Grammaire cognitive des prépositions : épistémologie et applications », Corela [En ligne], HS-22 | 2017, mis en ligne le 31 août 2017, consulté le 20 avril 2019. URL : http:// journals.openedition.org/corela/5003 ; DOI : 10.4000/corela.5003

Ce document a été généré automatiquement le 20 avril 2019

\section{(c) (i) (2)(2)}

Corela - cognition, représentation, langage est mis à disposition selon les termes de la licence Creative Commons Attribution - Pas d'Utilisation Commerciale - Partage dans les Mêmes Conditions 4.0 International. 


\title{
Grammaire cognitive des prépositions : épistémologie et applications
}

\author{
Jean-Rémi Lapaire
}

\section{Introduction}

1 Convoquer la grammaire cognitive pour rendre compte de l'emploi des prépositions en anglais n'est pas un acte anodin. Ce qu'on désigne par Cognitive Grammar s'est d'abord appelé Space Grammar (Langacker, 1982) et a très tôt accordé à l'espace (perçu, conçu, sémiotisé) un statut privilégié dans les langues et la cognition humaines ${ }^{1}$. Outils par excellence de localisation (concrète ou abstraite, littérale ou métaphorique), les prépositions ne peuvent que solliciter l'attention des linguistes cognitivistes, qui font de la cognition spatiale le pivot de la cognition générale.

2 La grammaire cognitive n'opère pas seule: elle est affiliée à la grammaire des constructions, à la sémantique cognitive, aux théories cognitives de la grammaticalisation, de la métaphore et de la métonymie, à la théorie des espaces mentaux et de l'intégration conceptuelle. Le dialogue interne avec ces disciplines est constant, comme en témoignent les rappels théoriques liminaires systématiquement effectués par Langacker dans ses écrits. Etre grammairien cognitiviste, c'est d'abord rejeter le principe d'une syntaxe autonome, déconnectée du sens et du milieu sociophysique. C'est aussi et surtout concevoir la grammaire comme un système symbolique à part entière, au même titre que le lexique ${ }^{2}$. Loin d'être vides ou arbitraires, les formes grammaticales sont considérées comme signifiantes (contentful, meaningful) et motivées ( motivated). Les morphèmes fonctionnels ne sont donc pas réductibles au statut d'outils ou d'utilitaires de la langue. Ce sont des unités symboliques à part entière, qui participent à la création «d'assemblages complexes» (complex symbolic assemblies) (Langacker, 2008 : 24). Ces assemblages, qui prennent souvent la forme de phrases, sont analysables comme 
autant « d'agencements conceptuels » (conceptual arrangements) ou de «scènes » (scenes). Dans le corpus (Atwood, 2000) ${ }^{3}$ nous rencontrons par exemple :

- des scènes d'action physique : My sister Laura drove a car off a bridge... she was rolling out pie dough on the white enamel kitchen table... I went upstairs to change... I opened the drawer...

- des scènes d'action verbale : I was informed of the accident by a policeman... I could barely get the words out...

- des scènes d'action mentale (reconnaître, se souvenir, analyser littéralement ou métaphoriquement un objet de conception) : I remembered Reenie from when we were little... I suppose... It wasn't the brakes, I thought... She was washing her hands of me...

- des scènes perceptuelles: They'd noticed her hands on the wheels... I could picture the smooth oval of Laura's face... I saw the notebooks...

4 Chacune de ces scènes fournit des éléments de "cadre » ou de " décor " (setting), dans lesquels prennent place des «actants» (participants). Un rapport «fond-figure » (figureground organization) est fréquemment établi entre ces diverses entités. L'énonciateur ( speaker) se comporte ainsi comme un observateur-conceptualisateur (viewer-cognizer) qui rapporte ce qu'il «voit» et "conçoit» (construe) sous divers "angles» (perspectives, vantage points) (Langacker, 2000 : 206-212). L'ensemble de cette organisation scénique est appelé stage model (Langacker, 2000: 42). Les prépositions y jouent un rôle majeur, puisqu'elles sont systématiquement sollicitées pour fournir des éléments de cadre ou de fond (ex. I ought to warn Richard, at his office), tout en ayant la capacité d'introduire ou de spécifier des actants ou figures (ex. I was informed of the accident by a policeman; To visit the morgue I would need gloves, and a hat with a veil).

5 Comment ces prépositions sont-elles caractérisées en tant qu'unités symboliques par la grammaire cognitive ? Pour répondre à cette interrogation, nous proposons un parcours menant du large à l'étroit. Nous commençons par une caractérisation générale de la grammaire cognitive et effectuons un bref retour sur ses fondamentaux. Nous abordons ensuite la question centrale de l'espace et de la cognition spatiale en grammaire cognitive avant de nous attacher aux prépositions proprement dites.

\section{Grammaire cognitive : principes fondateurs}

C'est en 1987, avec la publication du premier volume de Foundations que Langacker impose à la communauté scientifique une dénomination, cognitive grammar, et un cadre: theoretical prerequisites. À l'évidence, Langacker n'est pas seul impliqué dans l'entreprise. La linguistique cognitive, à laquelle se rattache nommément sa grammaire, a graduellement émergé durant les années 1960-70 sur la côte ouest des Etats-Unis, parmi des sémanticiens générativistes. La parution de Metaphors We Live By (Lakoff \& Johnson 1980) précipite les événements. Langacker publie peu après un article intitulé «Space Grammar, Analysability, and the English Passive» (Langacker, 1982) qui a un effet d'amorçage ${ }^{4}$. Dans les dix années qui suivent, il se consacre à la rédaction des deux épais volumes (théorique et applicatif) de Foundations (1987, 1991a). Mais ce sont surtout les recueils d'articles intitulés Concept Image and Symbol (1991b) et Grammar and Conceptualization (2000) qui assureront la diffusion internationale du modèle. Les textes qu'on y découvre sont issus de communications orales et s'avèrent plus accessibles que les pages un peu trop denses de Foundations. Conscient de la nécessité d'offrir une présentation unifiée et, espère-t-il, simplifiée de son modèle, Langacker publie en 2008 
une "introduction élémentaire» (basic introduction) à la grammaire cognitive. La simplification n'est guère évidente pour les néophytes. Mais le pari de la synthèse et de l'unification est tenu : le théoricien y réaffirme ses fondamentaux, inchangés depuis 1987, et les revisite avec un recul critique intéressant. Non sans humour, Langacker $(2008: 12)$ prend acte du succès grandissant de sa théorie mais aussi des dérives, incompréhensions et simplifications dont celle-ci a pu être l'objet :

From a limited exposure to CG, many people receive the impression that it is 'easy', apparently basing their assessment on its intuitive naturalness, its focus on meaning, the liberal use of diagrams, and the seeming absence of constraints. I agree at least in part: it is quite easy to do CG badly, and not so hard to do it indifferently. To do it well is obviously much harder.

7 De façon significative, Langacker ouvre son « introduction » (2008:3) avec des remarques sur «la grammaire et la vie» (grammar and life). Etre grammairien, c'est être un observateur du vivant et de la parole sociale. La grammaire n'est pas un système qui tourne sur lui-même ${ }^{5}$. Toutes les formes grammaticales sont « signifiantes » (meaningful) ${ }^{6}$ et encodent des processus de représentation (conceptualization, construal operations). La grammaire comme le lexique, a une "assise conceptuelle» (conceptual basis). Ses marqueurs et ses agencements (constructions) sont des unités symboliques à part entière : « lexicon and grammar form a gradation consisting soleley in assemblies of symbolic structures.» (Langacker, 2008: 5). Proposition «scandaleuse » (an outrageous proposal) pour ses détracteurs, « ascétisme théorique » (theoretical austerity) assumé pour Langacker et ses disciples (2008: 15). Le programme de la grammaire cognitive se définit donc comme minimaliste :

Let us define a symbol as the pairing between a semantic structure and a phonological structure, such that one is able to evoke the other (...) Grammar is concerned with how such elements combine to form complex expressions. The basic tenet of CG is that nothing beyond symbolic structures need to be invoked for the proper characterization of complex expressions and the patterns they instantiate. (2008:15)

8 Les « assemblages de symboles» (symbolic assemblies) que réalise la grammaire participent à la fois de la "fonction sémiologique» (semiological function) et de la «fonction interactionnelle " (interactive function) du langage. La dépendance étroite que Langacker établit entre formes lexico-grammaticales et fonctions langagières le conduit à rattacher l'approche cognitive au courant fonctionnaliste : « Language is shaped and constrained by the functions it serves » (2008: 7). Ainsi, le fait qu'il existe des marqueurs de localisation ( locative expressions) dans toutes les langues s'expliquerait par le besoin de représentation et d'expression de l'expérience spatio-temporelle dans les sociétés humaines, ni plus ni moins. Chaque langue soumettrait les marquages à un ensemble de "conventions " sémiologiques qui seraient «assimilées» (internalized) par les locuteurs. Des locuteurs qui, contrairement à ce que proclament les générativistes, ne créent pas en permanence de nouveaux énoncés à partir d'un nombre fini de règles de composition, mais puisent dans "un répertoire de constructions pré-fabriquées » (an inventory of prefabricated expressions, $2008: 19$ ). Le corpus (Atwood, 2000) n'échappe pas à cette règle : l'originalité stylistique et l'invention romanesque prennent sans cesse appui sur des constructions syntaxiques et des structures événementielles déjà installées dans l'usage. La consultation du COCA ( Corpus of Contemporary American English, 1990-2012), riche de plus de 450 millions de mots, confirme d'ailleurs que le canevas sur lequel la romancière brode est bien celui de la langue et de la cognition ordinaires. Un jeu d'échos, d'imitations et d'importations se joue 
en permanence sous le regard du lecteur: plunged off the bridge ${ }^{7}$, sailed off the bridges, stepping off a curb ${ }^{9}$; blowing around my head ${ }^{10}$. Même une construction plus poétique comme in the afternoon daylight est reliée à un répertoire de constructions du même type (IN + moment / qualité + daylight), inscrit dans l'usage contemporain de la langue anglaise ${ }^{11}$. Une conséquence importante de ce principe est qu'il ne suffit pas de s'interroger sur la valeur d'emploi de telle ou telle préposition dans un énoncé : il faut se demander à quelle construction (ou répertoire de constructions) elle se rattache.

Conscient des questionnements que suscite l'accolement de l'adjectif «cognitif » à " grammaire », Langacker (2008) assume ses responsabilités épistémologiques et aborde sans détour le statut de la cognition. Pour lui, le langage fait partie intégrante de la cognition humaine. Il en est simultanément le produit, l'expression et le moteur : « Rather than constituting a distinct, self-contained entity (a separate " module» or " mental faculty»), language is viewed as an integral part of cognition. » (Langacker, $2008: 8$ ). Le langage est donc indissociable des capacités générales de perception, de mémorisation, de conceptualisation qui structurent la cognition. Pour Langacker, les mécanismes de conceptualisation sont à la base de la sémantique linguistique ${ }^{12}$ et mettent en jeu un ensemble d'aptitudes élémentaires (basic cognitive abilities): schématiser / abstraire, catégoriser, mettre en relation, ordonner et séquencer (Langacker, $2000: 2-3$ ). Une place privilégiée est accordée à la schématisation qui jouerait un rôle particulier dans le fonctionnement de la grammaire : la multiplicité des usages situés d'une même forme ( usage events) ne serait possible que parce que ces formes sont pourvues d'un certain "schématisme » (schematicity) à un "niveau supérieur d'abstraction" (a higher level of abstraction) (17). Les énonciativistes et les psychomécaniciens français ne peuvent qu'entrer en résonance avec la conception langackerienne. Ils sont eux-mêmes à la recherche de "valeurs centrales", "d'invariants abstraits », de "formes schématiques » censées assurer l'unité profonde, en langue, d'usages discursifs dispersés.

Plus que tout autre, le domaine prépositionnel est propice à la mise en évidence du "schématisme» des marqueurs grammaticaux ${ }^{13}$. En effet, les grammairiens (toutes théories confondues) ont pour habitude de tracer des formes schématiques pour représenter et unifier les sens concrets ou « idéalisés » d'une même préposition :

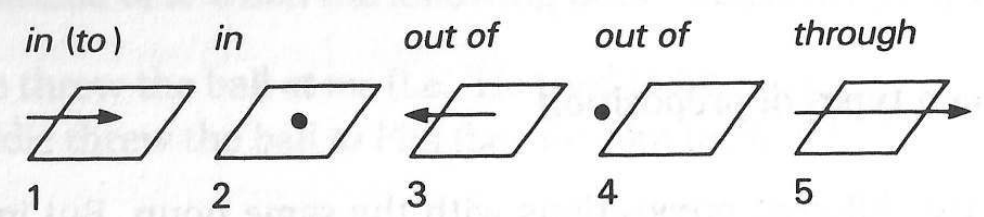

Figure 1 - IN, OUT, ON, THROUGH (Leech et Svartvik, 1994 : 93)

11 La production de schèmes visuels (pictorial diagrams) ne s'applique pas au seul schématisme des prépositions prises dans leur individualité. Il peut également concerner les configurations abstraites et idéalisées (configurational structures) partagées par diverses prépositions. Talmy (2000: 248) en fournit un exemple lorsqu'il figure le « vecteur du mouvement » commun à certains emplois de past, across, through, along et up (avec des verbes comme roll, sail, walk, run) de la façon suivante : 


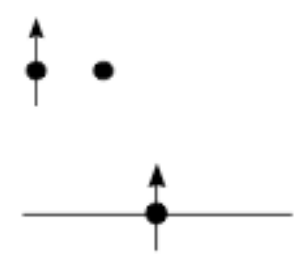

Figure 2 - IN et ON (Talmy $2000: 248$ )

The ball sailed past his head / ran across the border

Ces schèmes et configurations, obtenus par abstraction de l'expérience, accèdent au statut « d'archétypes conceptuels » (conceptual archetypes) ou de " primitives complexes » (complex primitives) en s'ancrant dans la cognition (entrenchment). Il peut s'agir aussi bien de configurations spatiales statiques que dynamiques.

Conceptual archetypes are experientially grounded concepts so frequent and fundamental in our everyday life that we tend to invoke them as anchors in constructing our mental world with all its richness and levels of abstraction. (...) I will cite just a few examples : a physical object, an object moving through space (...) a whole and its parts (...) walking, seeing something, saying something, holding something, handing something to someone. Also qualifying as archetypes are the functions of containment and support, as well as the factors involved in general orientation. (Langacker, $2010: 7$ )

Des scènes et configurations de ce type deviennent non seulement des « automatismes cognitifs» (cognitive routines) mais atteignent des degrés de décontextualisation permettant leur transfert métaphorique vers des domaines abstraits ${ }^{14}$, ou encore leur activation « simulée » ou « désengagée » (simulation, disengagement) (Langacker, $2010: 12$ ). Ainsi en est-il du placement ou du déplacenent d'entités à l'intérieur de... en direction de... ou hors d'un contenant. Dans Atwood (2000), nous lisons : she must have hidden (...) the stack of cheap school exercise books in the bureau drawer where I kept my stockings; I went into my dressing-room qui activent l'archétype du trajet effectué de l'extérieur vers l'intérieur d'un lieu-contenant (tiroir, pièce). Cet archétype a lui-même été obtenu par abstraction et généralisation d'un schème dynamique, élaboré à partir d'un ensemble récurrent de scènes spatiales de la vie ordinaire. On note qu'une métaphorisation de l'archétype est à l'œuvre dans I could barely get the words out. La cavité bucale est ici conçue comme un lieucontenant renfermant des mots-entités, susceptibles d'être expulsés vers l'allocutaire durant l'acte d'énoncation.

La modélisation de configurations spatiales (spatial arrangements) occupe une place importante dans la réflexion des grammairiens cognitivistes. Certains d'entre eux n'en restent pas là et s'intéressent à la genèse des marqueurs eux-mêmes : d'où proviennentils et que révèlent-ils du rapport corps, esprit, environnement? Bernd Heine apporte ici des éléments de réponse éclairants.

\section{Environnement, milieu ethnique et grammaire de l'espace}

Linguiste africaniste d'origine allemande, affilié aux courants typologiques, fonctionnalistes et cognitivistes, Heine s'intéresse à la genèse des systèmes grammaticaux (Heine, Claudi et Hünnemeyer, 1991; Heine et Kuteva, 2007). Le succès inattendu 
rencontré par un cycle de conférences donné en Australie en 1994 l'incite à rédiger un ouvrage de synthèse intitulé Cognitive Foundations of Grammar (1997) dans lequel il met en évidence les «stratégies de conceptualisation» (conceptualization strategies) à l'œuvre dans les langues pour « créer leur grammaires » (shape their grammars). Pour Heine, la création des formes langagières, lexicales ou grammaticales, est un processus historique, «motivé " de façon «interne» et "externe». Les mécanismes de conceptualisation sont indissociables de l'expérience socio-physique du monde et jouent le rôle de «force motrice majeure ${ }^{15} »$ dans l'élaboration de la sémiologie grammaticale :

Language structure is the product of our interaction with the world around us. The way we build discourses and develop linguistic categories can immediately be derived from the way we experience our environment and use that experience in species-specific communication » (Heine, $1997: 3$ )

Une illustration fournie par l'auteur est celle de l'opposition EST / OUEST. Heine rappelle que dans un très grand nombre de langues, les formes désignant ces points cardinaux renvoient originellement à l'idée de montée (ascension, émergence) ou de descente (déclin, chute, plongée) de l'astre solaire, en lien avec la perception naïve du mouvement céleste. Les jeux de la lumière solaire, croissante à l'aube et déclinante au crépuscule, peuvent également être invoqués pour nommer l'EST ou l'OUEST. Dans l'un et l'autre cas, l'expérience du milieu physique par un milieu ethnique particulier joue un rôle manifeste dans l'élaboration des formes linguistiques. Les choix sémiologiques opérés par une communauté, au cours de l'histoire, ne sont ni arbitraires ni mécaniques. Ces choix renvoient à des mécanismes collectifs de représentation qui, par définition, engagent la cognition :

Language structure, I argue, reflects patterns of human conceptualization because it is shaped by them. An approach that includes information on conceptual organization and conceptual transfer must have a higher explanatory potential than one that ignores such information. (Heine, $1997: 16$ )

Issu du courant typologiste (Typological Universal Grammar) et marqué par la grande tradition philologique allemande, Heine est un grammairien cognitiviste au sens fort, érudit et animé d'une évidente fibre comparatiste. Spécialiste reconnu des phénomènes de grammaticalisation ${ }^{16}$, il considère que pour décrire avec intelligence la «structure des langues » il faut commencer par se poser la question de l'« assise conceptuelle ${ }^{17}$ » des formes observées. Qu'on fasse ou non intervenir la diachronie, il est nécessaire d'identifier les systèmes de représentation et les mécanismes de conceptualisation qui modèlent la grammaire, sans jamais perdre de vue l'organisation sociale de la communication (rôles et identités, situations ritualisées d'interlocution) :

Although conceptualization strategies are perhaps the main driving force for linguistic categorization, conceptualization is not the only force that can be held responsible for why grammar is structured the way it is. Another, equally important force, is communication. (Heine, $1997: 14$ )

18 Heine articule de façon habile universalisme et relativisme : au-delà de leur dispersion géographique, ethnique et culturelle, les êtres humains possèdent le même « équipement intellectuel, perceptuel et physique ${ }^{18} »$ de base. C'est à partir d'un même répertoire, fini et identifiable, de stratégies de représentation (the same pool of options for conceptualization) qu'ils construiraient des systèmes linguistiques différents (14). Heine (1997: 11-12) cite à point nommé l'exemple des « repérages spatiaux » (spatial orientation) et note qu'il existe quatre grands scénarios de repérage qui transparaissent dans la genèse des formes et dans leur usage : 
- orientation par rapport à l'énonciateur ou à son partenaire (speaker-deictic orientation) $)^{19}$;

- orientation par rapport à un objet (object-deictic orientation $)^{20}$;

- orientation par rapport à un point de référence culturellement saillant (comme une montagne, un fleuve) (landmark orientation);

- ou alors repérage dans l'absolu (cardinal orientation) ${ }^{21}$.

De façon significative, Heine consacre un chapitre entier de Cognitive Foundations of Grammar (1997 : 34-65) à « la grammaire de l'orientation spatiale » (the grammar of spatial orientation). Outre ses propres travaux sur les langues africaines, sa principale source d'inspiration est la "grammaire de l'espace" (grammar of space) de Svorou (1994). Officiellement rattachée à la typologie linguistique (linguistic typology), mais résolument ancrée dans le paradigme cognitiviste, cette monographie connut un succès considérable lors de sa parution. Plus rarement citée aujourd'hui, elle demeure une référence incontournable pour tous les grammairiens cognitivistes.

Dans son étude, Svorou adopte le terme générique de spatial gram («marqueur spatial») pour transcender la spécificité morpho-syntaxique des 26 langues "non apparentées » qu'elle compare (26 genetically unrelated randomly selected langages): prépositions, adverbes, particules, localisateurs de toute catégorie sont regroupés sous une même étiquette. La chercheuse estime, à l'instar de Heine, que les formes que se donnent ces langues en général, et les " configurations spatiales » qu'elles adoptent en particulier ( spatial arrangements), sont tributaires de l'interaction des locuteurs avec leur environnement physique et de leurs "systèmes de croyances» (belief systems). La grammaire des langues est posée, dans ses fondements, comme une ethno-grammaire du " monde physique ». Ce dernier, vécu et conçu par les humains, engendre un ensemble de représentations collectives, qui trouvent une expression sémiologique conventionnelle dans chaque langue. Bien que les formes et les usages diffèrent, l'acte conceptuel fondamental qui consiste à « localiser » un être, un objet, un événement par rapport à une entité-repère semble universel. Un élément de « relativité » est ainsi introduit : un terme est situé par rapport à un autre terme, par rapport à une situation ou par rapport à un observateur, ce qui crée de facto une "asymétrie » dans la relation. Svorou (1994:9) s'inscrit résolument dans la ligne d'analyse langackerienne. Pour elle, chaque agencement de marqueurs est révélateur d'une mise en perspective et d'un traitement conceptuel particulier des relations spatiales : a construal of spatial arrangement by the speaker.

21 À partir de ses propres observations (portant sur 125 langues africaines), complétées par d'autres études réalisées dans les années 1980 sur des langues amérindiennes et océaniennes, et en s'appuyant sur les synthèses réalisées par Svorou (1994), Heine identifie trois grands « modèles originels pour la référence et l'orientation dans l'espace » (common source models for spatial orientation):

22 1. le corps humain (modèle anthropomorphique) ou animal (modèle zoomorphique). 


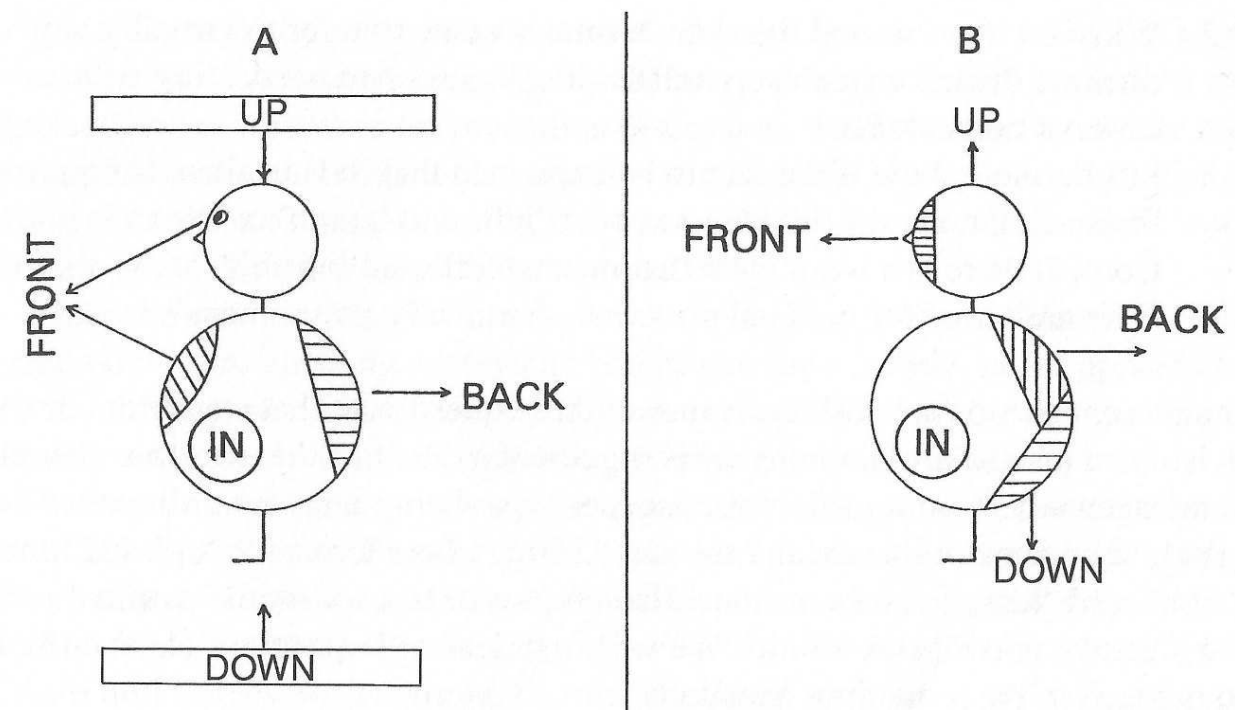

Figure 3 - Modèles corporels anthropomorphiques de structuration de l'espace : exemples du bantu et du nilotique occidental (Heine, $1997: 49$ )

2. les repères environnementaux perceptuellement et culturellement signifiants ( environmental landmarks) : par exemple, des termes dénotant à l'origine sky, heaven, summit pour UP ; earth, ground pour DOWN ; field, doorway pour FRONT ; track, trace pour BACK ; house, shore, land pour IN ; field, doorway pour OUT (39).

3. certaines notions dynamiques (dynamic concepts) : come, go, following, precede, pass.

En anglais, back ${ }^{22}$ et (in) front (off $)^{23}$ sont de bonnes illustrations du modèle corporel, down ${ }^{24}$ du modèle environnemental et preceding (pour signifier BEFORE) / following (pour signifier AFTER) du modèle verbal dynamique. Dans ce dernier cas, on note la prévalence d'une forme non finie du verbe (cf. le français « suivant », « précédent »).

Heine souligne la centralité du corps dans l'élaboration des repérages spatiaux ${ }^{25}$ et analyse la manière dont s'opèrent les projections conceptuelles parties du corps > parties d'objets > rapports topologiques (from body-part to spatial concept / relation).

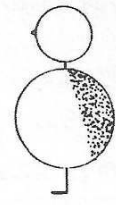

।

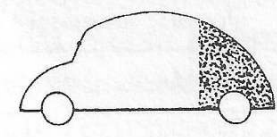

II

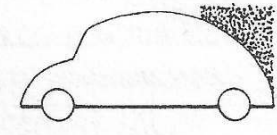

III
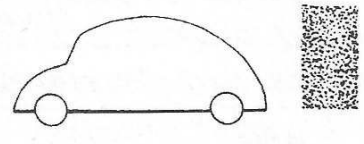

IV 


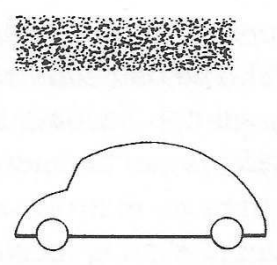

1

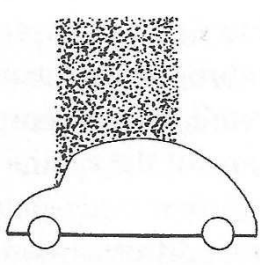

II

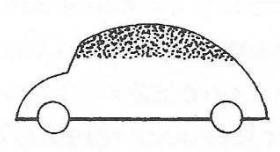

III

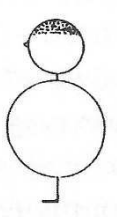

IV

Figure 5 - Ciel > au-dessus > partie supérieure d'un objet > dessus du crâne (Heine, $1997: 45$ ) africaines (Afri.) et océaniennes (Océa.) de son corpus, Heine extrait les généralisations suivantes :

31

- UP : la TÊTE domine nettement pour l'expression des UP-terms (87 \% Afri., 61 \% Océa.), devant le VISAGE, l'ÉPAULE, les CHEVEUX et le FRONT. Quelques langues du corpus africain, probablement influencées par le modèle zoomorphique, utilisent le Dos pour signifier ce qui est « en haut » ou « au dessus ».

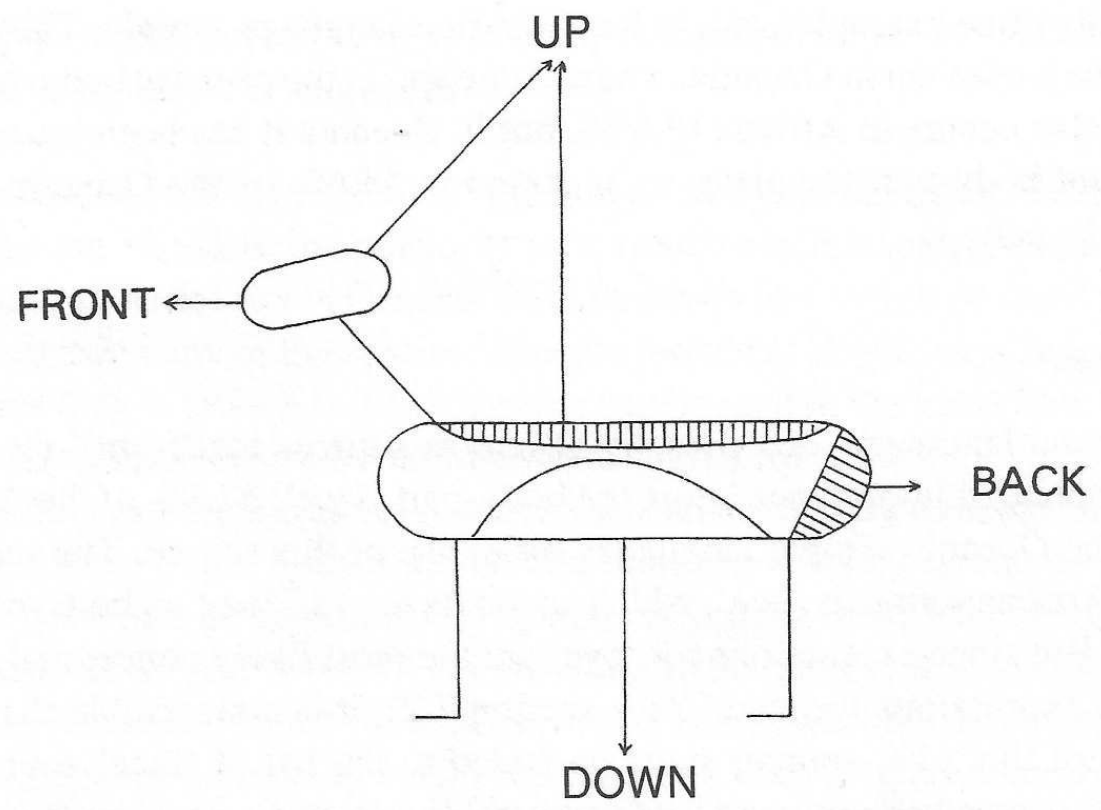

Figure 6 - Modèle zoomorphique prototypique (Heine, $1997: 41$ )

- FRONT : le VISAGE (la FACE) l'emporte très largement (52,8 \% Afri., 72,1 \% Océa.) pour exprimer ce qui est «devant». D’autres parties du corps peuvent être impliquées : les YEUX, la POITRINE, le FRONT et même le VENTRE pour certaines langues océaniennes ${ }^{27}$.

- BACK : sans surprise, le Dos s'avère dominant et d'une grande universalité (77 \% Afri., 
95 \% Océa.). L'ANUS et les FESSIERS jouent cependant un rôle non-négligeable dans les langues africaines du corpus considéré (22,3\% Afri.).

- IN : le schéma corporel est ici encore très sollicité. En Afrique, le VENTRE est de loin la zone la plus souvent invoquée ( $92,1 \%$ Afri.), avec de très marginales allusions à la PAUME (4,8\% Afri.) ou au CœUR (3,1\%). La situation est plus diversifiée dans les langues océaniennes du corpus: les DENTS (26.7\%), le VENTRE $(17.8 \%)$, le cœUR (13.3\%), le FOIE (11.1\%) and les INTESTINS (11.1\%).

- DOWN : l'environnement l'emporte ici sur le schéma corporel. Les éléments de type EARTH, GROUND sont très sollicités. Lorsque des parties du corps sont impliquées, l'ANUS et les FESSIERS sont privilégiés dans les langues africaines, le PIED ou la JAMBE dans les langues océaniennes.

Heine note par ailleurs que le processus de grammaticalisation, une fois engagé, fait évoluer morphosyntaxiquement les noms désignant originellement des parties du corps ou des repères environnementaux. Le passage se fait le plus souvent du nominal vers l'adverbial. Intégrés à la sphère grammaticale et spécialisés dans l'expression de rapports spatiaux, les ex-noms acquièrent les propriétés formelles et sémantiques d'adverbes de lieu, de prépositions, de postpositions, de particules...

On le voit, le cognitivisme de Heine est un cognitivisme des fondements (cf. le titre de son ouvrage), sensible aux origines et au milieu ethnique, plus incarné et socialisé que celui de Langacker car immergé dans l'oralité des langues et des cultures africaines. Tout en privilégiant la dimension idéationnelle et représentationnelle du langage, Heine ne fait jamais l'impasse sur sa fonction communicative ${ }^{28}$. Il est important de le souligner car trop de linguistes cognitivistes ont pris l'habitude d'interroger des corpus électroniques en s'affranchissant du milieu, des situations et de l'interaction. Comme Givòn (2002), mais avec plus de méthode et sans faire intervenir de composant bio-évolutionniste, Heine est partisan d'une grammaire que nous pourrions appeler « cognitive-fonctionnelle ». Dans cette approche liant forme et fonction, les mécanismes de représentation, les formes langagières et les fonctions expressives sont totalement intégrés et solidaires. La morphologie de la langue, lexicale et grammaticale, dépend directement de sa fonction organisatrice et régulatrice des rapports inter-individuels. Ces rapports sont dynamiques. Ces rapports sont une interaction au sens fort: interaction (physique, mentale) avec le monde, interaction (physique, mentale, sociale) avec les autres. In fine, la grammaire cognitive de Heine s'impose comme une « ethno-grammaire cognitive-interactionnelle. »

\section{Les prépositions dans une grammaire cognitive de l'anglais}

Tracer et interpréter l'origine des marqueurs spatiaux, examiner dans quelle mesure ces marqueurs révèlent les mécanismes de structuration de la cognition spatiale a toujours intéressé les chercheurs rattachés à la mouvance cognitiviste. Mais cette recherche, à orientation diachronique et typologique, a cessé d'être un axe d'investigation privilégié en grammaire cognitive. Tout se passe comme si la synthèse critique finale des recherches intenses menées dans les années 1980-90, brillamment opérée par Levinson (2004), avait permis d'asseoir et de régler une fois pour toute la question du rapport fondateur à l'espace dans l'expérience et la cognition humaines : 
Spatial cognition is at the heart of our thinking. It has long been noted that spatial thinking provides us with analogies and tools for understanding in other domains, as shown by the efficacy of diagrams, the pervasive spatial metaphors of everyday language, the evocativeness of place in memory, and the special role that geometry, astronomy and cartography have played in the development of science and technology. (Levinson, 2004 : xvii). de la cognition générale ou seulement l'un de ses piliers? Comment s'articulent la cognition individuelle des sujets et la cognition générique, collective dont est porteuse la langue ? À quel niveau se situe la cognition révélée par la sémiologie langagière ? Alors que ces questions apparaissaient comme brûlantes et essentielles dans les moments premiers de la linguistique cognitive (Lakoff et Johnson, 1980, 1999, Lakoff, 1987, Johnson, 1987), les grammairiens cognitivistes s'en sont progressivement éloignés, pour privilégier un deuxième questionnement : pourquoi et comment les marqueurs spatiaux évoluent-ils vers des sens et usages "non spatiaux» (Evans, 2010)? À l'évidence, les théories cognitives de la métaphore, de la métonymie, de l'intégration conceptuelle auxquelles ont été exposés tous les grammairiens cognitivistes et qui constituent leur patrimoine identitaire, encouragent ce type d'interrogation. Mécanismes de projection, d'association ou de mélange inter-domaines sont assurés d'être au rendez-vous.

Dans ce contexte, Cognitive English Grammar de Radden et Dirven (2007) mérite une attention particulière car c'est la première grammaire cognitive entièrement dédiée à la langue anglaise. Les auteurs optent pour des définitions et des analyses consensuelles qui fournissent un aperçu assez fidèle des positions partagées par la communauté. A défaut d'être tout à faire homogènes, ces positions sont restées stables sur les fondamentaux (origine, motivation et sémantisme des formes, lien organique entre sémiologie grammaticale et cognition générale). Il faut néanmoins signaler que la perspective est appelée à prendre un tour plus syntaxique, sous la pression grandissante des usage-based models. Ces derniers, qui se réclament de la grammaire des constructions (Fillmore, 1988, Goldberg, 1995, Croft, 2001), sont attentifs à la distribution effective des items dans le discours. Ils s'appuient sur de vastes corpus et des méthodes quantitatives avancées (Glynn, 2010) pour identifier la nature, la fréquence d'occurrence et le degré de prévisibilité des collocations et des constructions. L'idée centrale est que le degré d'exposition (input frequency) aurait un impact décisif sur l'acquisition, la reconnaissance, l'interprétation, l'appropriation et le réemploi des formes: "Frequency does not only affect the storage of words, it also plays a role in the entrenchment of grammatical patterns (...) processing factors (...) and the formation of abstract schemata" (Hoffman, 2011 : 5-6). Ainsi, les structures ne seraient pas générées sur un mode compositionnel, au moyen de règles abstraites d'assemblage, mais obtenues par analogie avec des constructions pré-existantes, au gré de variations individuelles et situationnelles. Les constructions effectivement employées par les locuteurs iraient de l'idiome figé, reproduit tel quel (ex. lost in translation), à l'agencement totalement ouvert lexicalement, en passant par la collocation (ex. lost + in $+\mathrm{SN}>$ lost in [his] thought [s], lost in space, in the forest, in the fog, in the wilderness, in the forest, in the desert, in a maze, in the mail, in a reverie, in contemplation $\left.{ }^{29} . ..\right)$. Le locuteur ne partirait donc pas d'items à combiner mais de patterns pré-établis. Il procèderait par détournements, réinvestissements, généralisations de « matière langagière concrète ${ }^{30}$ » déjà présente et attestée par l'usage.

Nous n'en dirons pas davantage sur la grammaire des constructions et la place grandissante que cette dernière occupe au sein des grammaires cognitives. Nous nous en 
tiendrons ici à l'approche plus sémantique de Radden et Dirven (2007). Leur étude des prépositions occupe l'essentiel du chapitre intitulé "Space and extensions of space" (303-334). Le rapport à l'espace et à la matière est donc posé de façon explicite comme une donnée fondatrice, en conformité avec la conception qu'entretiennent la majorité des linguistes cognitivistes sur l'articulation entre sémiologie langagière et expérience sociophysique du monde. La grammaire cognitive, nous l'avons déjà signalé, s'est d'abord appelée space grammar (Langacker, 1982) avant d'être rebaptisée cognitive grammar (Langacker, 1987). Pourquoi space? Parce que l'espace physique (physical space) et les entités ou subdivisions qui lui sont associées dans la cognition ordinaire fournissent un socle empirique pour rendre compte des mécanismes de symbolisation portés par le lexique et la grammaire. En mobilisant les idées de «domaine » (domain), de « région » ( region), de «zone " (zone), de "repère » (landmark), de «figure " (figure), de «fond " ( ground), il devient possible de forger les notions d'« espace abstrait» (abstract space), d'« espace sémantique » (semantic space), d'« espace épistémique » (espistemic space), de "domaine cognitif» (cognitive domain), de «zone active» (active zone), et de mettre en place l'opposition centrale «figure / fond» (figure/ground), elle-même à la base de la distinction-clé « cible [ou trajecteur] / site [ou point de repère] » (trajector / landmark).

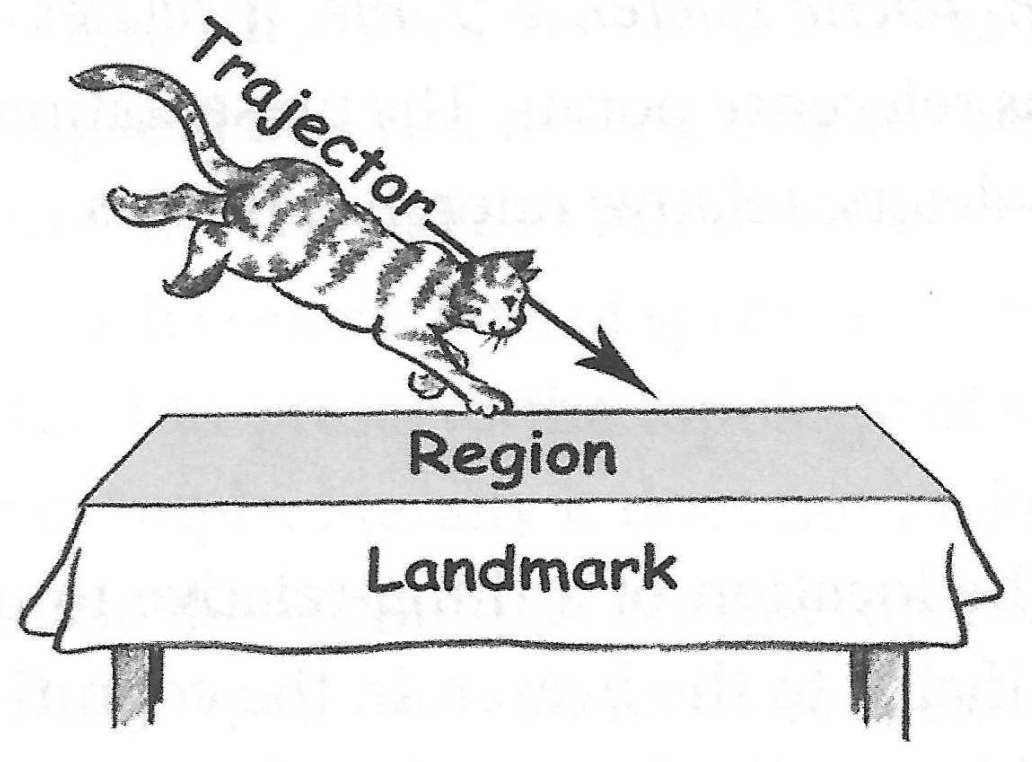

Figure 7 - The cat jumped on the table (Radden \& Dirven, 2007 : 306)

Radden et Dirven prennent appui sur le cadre théorique construit par Langacker (1982, 1987, 1991a, 1991b, 2000, 2008), qu'ils croisent avec la théorie cognitive de la métaphore de Lakoff et Johnson $(1980,1999)$. Ils invoquent également « une topologie de l'espace » ( topology of space) qui serait ancrée dans notre rapport ordinaire à «l'espace physique » ( physical space) (307) et « reflétée dans la langue ». La mobilisation de l'expérience et de la cognition ordinaires pour rendre compte de l'usage grammatical, nous l'avons vu, est un trait marquant et constant de l'approche cognitive. Pareille référence à l'expérience de l'univers peut surprendre et ne manquera pas d'agacer les adeptes de cadres plus formels, attachés à un traitement autonome et modulaire de la grammaire. La démarche n'en demeure pas moins légitime en interne, au sein du paradigme cognitiviste. La sémiologie grammaticale, qui permet d'articuler la pensée et son expression verbale, fait partie 
intégrante de la sémiologie langagière. Or toute sémiologie est liée dans ses fondements à l'expérience de l'univers et aux représentations ethniques qui en découlent (Heine, 1997). La « topologie » (naïve) dont il est question chez Radden et Dirven (2007), intègre donc à l'analyse des marqueurs la perception physico-culturelle du monde et les catégories d'entendement qui en découlent. Sont principalement convoquées :

- les notions de "localisation, direction et extension" (308), qui elles-mêmes se combinent avec les paramètres «statique » et « dynamique »;

- les idées de «lieu » (place), «point de départ» (source), «destination, point d'arrivée, but » (goal), « chemin, trajectoire » (path), « orientation » (orientation);

- la « dimension 0, 1, 2, 3 » (zero, one, two, three-dimensionality).

Table 12.3. The basic dimensional prepositions of English

\begin{tabular}{|c|c|c|c|c|}
\hline \multirow[b]{2}{*}{ dimensions } & \multirow{2}{*}{ location } & \multicolumn{3}{|c|}{ direction } \\
\hline & & SOURCE & GOAL & PATH \\
\hline $\begin{array}{l}\text { 0-dimensional } \\
\text { POINT }\end{array}$ & $\begin{array}{l}\text { at, by, near, } \\
\text { close to, with }\end{array}$ & $\begin{array}{l}\text { from, } \\
\text { away from }\end{array}$ & $\begin{array}{l}\text { to, at, for, } \\
\text { towards }\end{array}$ & by, past, via \\
\hline $\begin{array}{l}\text { 1- \& 2-dimensional } \\
\text { LINE / SURFACE }\end{array}$ & on, on top of & off (of) & on(to), against & $\begin{array}{l}\text { along, about, } \\
\text { around }\end{array}$ \\
\hline $\begin{array}{l}\text { 3-dimensional } \\
\text { CONTAINMENT }\end{array}$ & $\begin{array}{l}\text { in, within, inside, } \\
\text { between, among }\end{array}$ & $\begin{array}{l}\text { out of, } \\
\text { outside of }\end{array}$ & in $($ to $)$ & $\begin{array}{l}\text { through, } \\
\text { throughout }\end{array}$ \\
\hline
\end{tabular}

Figure 8 - Les prépositions dimensionnelles de base (Radden \& Dirven, 2007 : 310)

41 Le corpus (Atwood, 2000) contient des exemples qui illustrent bien ce que Radden et Dirven (2007) nomment " prépositions topologiques » ou « dimensionnelles » (topological or dimensional prepositions) : I ought to warn Richard at his office (point); A hot wind was blowing around my head (lignes / volutes); She'd sit us (...) on the white enamel kitchen table (surface); ink spilled in water (contenant). Mais le texte renferme également des prépositions impliquées dans des processus d'association et de complémentation, ne faisant pas a priori intervenir d'élément topologique ou dimensionnel, notamment of ( What had she been thinking of? She was washing her hands of me. Of all of us) et with (my entire face was rigid with pain).

La grammaire cognitive, nous l'avons vu, traite les situations comme autant de « scènes » (scenes) ou « configurations événementielles » (events) que la syntaxe ordonne en fonction de la perspective / conception du locuteur (construal). À chaque construction linguistique correspond ainsi une conceptualisation ou «image» différente, ce que résume l'expression grammar as image, employée par Langacker (1991b : 12)

Grammar as image. Like lexicon, grammar provides for the structuring and symbolization of conceptual content, and is thus imagic in character. When we use a particular construction or grammatical morpheme, we thereby select a particular image to structure the conceived situation for communicative purposes (...) The symbolic resources of a language generally provide an array of alternative images for describing a given scene.

L'imagerie grammaticale procède non par reflet direct mais par mise en " perspective ", comme le souligne Vandeloise (1986) : « les objets (localisés) ne sont pas analysés avec la rigueur d'une épure mais idéalisés en fonction de la perspective selon laquelle ils sont considérés. » (45). L'idéalisation tient au fait que la réalité matérielle et la perception sont retravaillées, réagencées par l'esprit humain avec une grande « liberté ». Le plus souvent, 
ce travail aboutit à une forme de schématisation, calibrée et encadrée par les conventions sémiologiques de la langue :

La liberté avec laquelle nous modelons et transformons la réalité objective (est) énorme. L'idéalisation d'un objet peut se réduire, par métonymie, à une de ses parties. Elle peut encore désigner son contour idéalisé, dont ont été gommés les détails superflus, ou l'approximation géométrique la plus proche (...) Enfin, un objet peut être représenté dans une relation spatiale par ses projections horizontale et verticale (ou encore) la réduction à un point. (Vandeloise, $1986: 48-49$ )

Ainsi, la réalité physique du bureau de Richard (Atwood, 2000) est-elle, au départ, celle d'un espace tridimensionnel. Mais dans le discours, cet espace est soumis à un reparamétrage : il est « réduit à un point ", sans surface, sans profondeur (at his office). De même, le pont (bridge), qui joue un rôle central dans l'épisode, est-il matériellement un ouvrage d'art volumineux. Or seule la partie superficielle du tablier de ce pont est utilisée comme zone active ${ }^{31}$ dans le récit de l'accident. Lorsque nous lisons bridge, nous comprenons, par métonymie, la chaussée du pont. Celle-ci semble privée de toute épaisseur et avoir été réduite à une surface horizontale. De la même façon, la zone active $\mathrm{du}$ véhicule (car) est celle des pneumatiques, qui en temps ordinaire, adhèrent à la chaussée. On voit donc que la scène physique de l'accident, qui semble décrite avec minutie et réalisme, est en fait idéalisée et simplifiée, sur un mode métonymique. La préposition off, qui marque ici une rupture de contact entre deux surfaces, atteste de ce remodelage et de la «transformation de la réalité objective » : my sister Laura drove a car off a bridge; the car sailed off the bridge. Le reparamétrage des critères dimensionnels $(3>2)$ permet une schématisation de l'expérience, comme le confirmerait certainement l'observation de la gestualité co-verbale, si nous avions été en présence d'une narration orale. En synchronie avec la parole, les mains auraient certainement tracé des gestes directionnels de type iconique (dans la classification de McNeill, 1992). Par exemple, la paume de la main dominante se serait d'abord frottée contre la paume de l'autre main dans l'axe sagital, pour évoquer le contact dynamique des roues avec la surface du pont. Puis les doigts de la main dominante se seraient écartés vers l'extérieur, afin d'amorcer la déviation de trajectoire, avant que les paumes ne se séparent (pour indiquer la rupture de contact). Ce type de gestualité iconique est à la fois concret et abstrait, descriptif et sélectif. Tout en donnant l'illusion de " coller au réel », l'esprit sélectionne quelques traits pertinents, pour rendre saillant tel ou tel aspect d'une scène. Arnheim (1969), dans Visual Thinking, a très bien caractérisé ce phénomène. L'abstraction de schèmes, à partir d'une réalité perceptuelle riche et multisensorielle, est le fondement de l'abstraction :

Man, in perceiving the complex shapes of nature, creates for himself simple shapes, easy on the senses and comprehensible to the mind. One function of these shapes is that of producing physical equivalents of non-mimetic images harbored by the mind. (216)

La schématisation des événements effectuée grâce à la conceptualisation langagière facilite leur mise en parallèle avec d'autres scènes. À preuve, la mise en équivalence de la rupture roues de la voiture / chaussée du pont avec la rupture semelles du piéton / surface d'un trottoir dans (Atwood, 2000) : Laura had turned the car sharply and deliberately, and had plunged off the bridge with no more fuss than stepping off a curb. Bien qu'elles soient dramatiquement distinctes (acteurs, circonstances, conséquences), les deux scènes convergent au niveau plus abstrait de leur schématisme : rupture de contact, dans le plan horizontal, entre un objet et son support. L'identité structurale $\mathrm{V}+$ off $+\mathrm{SN}$, permet de construire syntaxiquement ce parallèle et de l'activer conceptuellement. 

pour aborder les phénomènes d'extension métaphorique de sens spatiaux, matériels, concrets, etc. à des domaines de signification non-spatiaux, immatériels, abstraits. On rencontre ainsi, dans (Atwood, 2000), divers emplois de in + SN qui peuvent être placés sur le gradient de dé-substantiation suivant: ink spilled in water (matière liquide); my hair lifting and swirling in (the) hot wind (matière gazeuse); a shirtwaist with a small rounded collar, in a sober colour-navy blue or steel grey or hospital-corridor green; I must be in shock. En grammaire cognitive, ces emplois doivent nécessairement pouvoir être reliés les uns aux autres. Un traitement atomisé et homonymique est par principe exclu. Radden et Dirven (2007) adhèrent à ce principe mais ne vont jamais au-delà de la projection métaphorique inter-domaine (cross domain mapping) ou de l'activation de schèmes-images (image schemata), dans la pure tradition lakoffienne et johsonienne. Ici, l'analyse qu'ils proposeraient se ramènerait simplement à la formule : EMOTIONS, SENSATIONS, PERCEPTIONS ARE [COGNIZED, EXPERIENCED or CONSTRUED AS] CONTAINERS (cf. IN navy-blue, grey, green; IN shock).

Ce type d'analyse diffère de la méthode initialement proposée par Vandeloise (1986: 71-72). Ce dernier postule l'existence d'une « impulsion » première, qu'il définit comme le «nœud par lequel s'effectue le premier accès au réseau de significations (d'un mot ou d'un marqueur)». À partir de cette «impulsion» (qui s'exprime sous la forme d'un «concept global»), se développeraient une "multiplicité de règles d'usage et de caractéristiques » ainsi que "des restrictions de sélection». Identifier l'impulsion puis tracer les développements revient à pratiquer ce que Vandeloise appelle une « diachronie logique ». Radden et Dirven (2007) ne s'y hasardent pas. Par facilité, peut-être, ils préfèrent adapter la sémantique cognitive de Lakoff et Johnson à la grammaire. L'interaction avec le monde matériel, les positionnements et les déplacements dans l'espace, y sont conçus comme fondateurs de l'expérience et de la cognition humaines (Lakoff et Johnson, 1980). À ce titre, le corps, la matière et l'espace sont pourvoyeurs de notions et schèmes à projeter métaphoriquement sur d'autres domaines, réputés plus abstraits.

Space is one of the most basic and tangible domains of experience. It is characterized by an elaborate system of spatial relations. By metaphorically extending the senses of spatial prepositions, the domain of time and other abstract domains are conceived of and expressed in a variety of space-like ways. The analysis of prepositions of space and their motivated extensions amply demonstrates the pervasiveness of conceptual metaphor in grammar. (Radden \& Dirven, 2007 : 303)

Tout comme le lexique, la grammaire est le produit sémiologique de ce que Lakoff et Johnson (1999) appellent «raison incarnée " (embodied reason) ou encore «raison imageante » (imaginative rationality). Cette raison est largement inconsciente d'elle-même, de son fonctionnement et de son rayon d'action :

Most prepositions denote spatial relations as their basic and historically primary meanings, and their uses in abstract domains are metaphorical extensions of spatial meanings. Seeing and describing time, circumstances, cause, reason or purpose in terms of space is so natural that we have to think twice before we realise that we are dealing with metaphor. (Radden \& Dirven, 2007 : 304)

49

Il est tout à fait significatif que Radden et Dirven parlent « d'espace abstrait » (abstract space) pour se référer à des «domaines » (abstract domains) tels que la circonstance, la cause, la raison et le but ${ }^{32}$. Pourquoi maintenir coûte que coûte le terme «d'espace »? 
Pour les auteurs, qui ont été nourris à la sémantique cognitive de Lakoff et Johnson (1980, 1999) et à la théorie des espaces mentaux de Fauconnier et Turner (2002), il y a un lien phénoménologique évident entre la construction de l'abstraction (dans des espaces mentaux) et notre expérience sensori-motrice et mentale de l'espace (Arnheim, 1969). La grammaire en apporte une preuve, en autorisant des prépositions «topologiques » ou "dimensionnelles» comme in(to), on (top of), by, through, out of à investir métaphoriquement d'autres domaines de signification. Toutefois, ces domaines réputés plus «abstraits» sont également accessibles à partir de prépositions non-spatiales ou adimensionnelles (dans leur usage actuel), comme of ou with. Ainsi, dans my entire face was rigid with pain (Atwood, 2000), l'état dénoté par l'adjectif rigid est-il engendré par le complément prépositionnel with pain. L'association marquée par with, responsable de la lecture causale ( the pain caused the rigidity in my face »), doit-elle obligatoirement être ramenée à des primitives spatiales?

Nous ne le pensons pas mais restons attachés, aux côtés de Radden et Dirven (2007) au maintien du terme "espace». Nos raisons sont cependant différentes des leurs et la terminologie que nous préconisons n'est pas la même. Ainsi préférons-nous parler «d'espace épistémique » (epistemic space) ou "d'espace de conceptualisation » (conceptual space) plutôt que « d'espace abstrait ». Ce type d'espace est de nature symbolique. À l'oral, il se confond avec l' « espace gestuel» (gesture space) des locuteurs, qui, pour raisonner, argumenter, expliquer, raconter placent et relient manuellement des entités invisibles devant eux (Lapaire, 2011). Calbris $(1990,2011)$ et McNeill $(1992,2005)$, ont depuis longtemps mis en évidence l'organisation spatiale de l'argumentation et du récit, dans la gestualité coverbale. Relier, enchaîner, unir, opposer, comparer, classer, etc. des idées ou des événements est une activité cognitive qui se traduit, au niveau du symbolisme phonogestuel, par une mise en espace dynamique.

51 Pour nous résumer, nous dirons que la présentation de Radden et Dirven (2007) illustre bien la thèse localiste, qui reste largement dominante en grammaire cognitive :

- primat du rapport à l'espace et des mécanismes de repérage pour comprendre l'origine et le développement des formes ;

52 - recours à des configurations spatiales, plus ou moins schématiques, pour modéliser le fonctionnement des unités impliquées.

On pourra regretter que l'approche de Radden et Dirven (2007) n'ait pas cherché à affiner ce « localisme » (Victorri, 2010) en intégrant davantage les travaux de Vandeloise (1984, 1986, 1991, 2006). Langacker lui-même $(2008,2010)$ reconnaît au linguiste belge une volonté marquée d'unifier les différents emplois d'une même préposition, en assignant à chacune d'elle une "configuration spatiale unique» (a single spatial configuration). Il souligne que pour parvenir à transcender la diversité des usages et des interprétations, Vandeloise procède à une "idéalisation géométrique " (geometric idealization). Cette démarche, estime Langacker, ne peut aboutir que si la configuration de la «valeur centrale » (central value) est suffisamment riche ou composite pour permettre de motiver ramifications et extensions. Vandeloise, nous l'avons vu, assigne à chaque préposition une "impulsion logique» à partir d'une "primitive complexe» (complex primitive): «primitive» car pré-linguistique et «complexe» car nécessitant plusieurs traits ou propositions unifiés pour être caractérisée. S'inspirant de la démarche de Vandeloise, Langacker propose pour in en anglais la «concavité » et l'« inclusion spatiale » comme primitives "configurationnelles"; le "rangement ", la "protection» et le "contrôle multidirectionnel », comme primitives « interactionnelles ». 

inspirée par Vandeloise, qui très tôt a perçu la nécessité d'assigner des propriétés fonctionnelles comme le rapport entre contenant (container) et contenu (content) ou encore entre charge (burden) et support (bearer). Dans The car fell a hundred feet into the ravine (...) and rolled down into the shallow creek at the bottom (Atwood, 2000), in assignerait à ravine et à creek la fonction de contenants (container) et confèrerait à la voiture le rôle de contenu (content). Dans They'd noticed her hands on the wheels, on donnerait une fonction de support / soutien (support, bearer) au volant, tandis que les mains auraient le statut de charge ou d'élément porté (burden). Le choix de la préposition serait fonction du rôle que les entités jouent l'une par rapport à l'autre, dans la perception et l'esprit de l'énonciateur. C'est ce rôle et non une disposition spatiale objective des éléments qui motiverait le choix. Victorri (2010) estime à juste titre que l'approche de Vandeloise s'affranchit clairement des naïvetés et facilités du «localisme objectiviste». Le « localisme enrichi » que Vandeloise pratique intègre des considérations syntaxiques tout en permettant de mettre en évidence des mécanismes cognitifs majeurs, comme "l'anticipation", perceptible dans des énoncés aussi simples que Je vais à la poste, dans lequel l'énonciateur trace mentalement l'entier du trajet, non encore effectué au moment de l'énonciation. Mais Victorri (2010) là encore attire notre attention sur le fait que Vandeloise ne se dégage pas entièrement du localisme. Son étude du verbe aller et de sa complémentation (Vandeloise, 2007) ne parvient pas à se dégager de l'idée de «déplacement ", concret ou abstrait, alors qu'il aurait pu s'en passer, en invoquant l'engagement dynamique d'une scène et l'anticipation de son aboutissement, applicable aussi bien à des configurations spatiales (Yvan va à Prague) que non spatiales (Yvan va manger).

\section{Conclusion}

De la genèse des formes à leur intégration à des constructions, de l'expérience physique à l'expression linguistique, la grammaire cognitive propose une lecture décentrée, intégrative et enrichie de la sémiologie grammaticale. Le traitement particulier de la cognition spatiale et des prépositions donne un accès privilégié à l'ensemble de la démarche, qui, nous avons pu le voir, lie syntaxe et sémantique, symbolisme lexical et symbolisme grammatical, mécanismes de représentation et expression.

\section{BIBLIOGRAPHIE}

Arnheim R. (1969). Visual Thinking. Berkeley : University of California Press.

Atwood M. (2000). The Blind Assassin. Toronto, Canada : Emblem, McClelland \& Stewart.

Calbris G. (1990). The Semiotics of French Gestures. Bloomington : Indiana University Press.

Calbris G. (2011). Elements of Meaning in Gesture. Amsterdam / Philadelphia : John Benjamins. 
Croft W. (2001). Radical Construction Grammar : Syntactic theory in typological perspective. Oxford/ New York : Oxford University Press.

Evans V. (2010). "From the spatial to the non-spatial : the 'state' lexical concepts of in, on and at", in Evans V. et Chilton P. (eds.), Language, Cognition and Space. Sheffield : Equinox Publishing, 215-248.

Fauconnier G. et Turner M. (2002). The Way We Think. New York : Basic Books.

Fillmore C. (1988). "The Mechanisms of Construction Grammar". Berkeley Linguistics Society, vol. 14, 35-55.

Fortis J.-M. (2010). « De la grammaire générative à la grammaire cognitive : origine et formation de la théorie de Ronald Langacker ». Histoire Épistémologie Langage 32/II, 109-149.

Givòn T. (1993). English Grammar : A Function-Based Introduction. Volume 1. Amsterdam : John Benjamins.

Givòn T. (2002). Bio-linguistics : The Santa Barbara lectures. Amsterdam : John Benjamins.

Glynn D. (2010). “Corpus-Driven Cognitive Semantics. An introduction to the field”. In CorpusDriven Cognitive Semantics. Quantitative approaches. D. Glynn \& K. Fischer (eds), Berlin : Mouton de Gruyter, 1-42.

Goldberg A. (1995). Constructions : A Construction Grammar approach to argument structure. Chicago : University of Chicago Press.

Heine B. (1997). Cognitive Foundations of Grammar. Oxford : Oxford University Press.

Heine B, Ulrike C. et Hünnemeyer F. (1991). Grammaticalization : A Conceptual Framework. Chicago : Chicago University Press.

Heine B. and Kuteva T. (2007). The genesis of grammar : a reconstruction. Oxford : Oxford University Press.

Hoffmann T. (2011). Preposition placement in English. A usage-based approach. Cambridge : Cambridge University Press.

Hopper P. et Traugott E. Closs. (2003). Grammaticalization. Second Edition. Cambridge : Cambridge University Press.

Johnson, M. (1987). The Body in the Mind. Chicago : The University of Chicago Press.

Lakoff J. (1987). Women, Fire and Dangerous Things. Chicago : The University of Chicago Press.

Lakoff G. et Johnson M. (1980). Metaphors We Live By. Chicago : The University of Chicago Press.

Lakoff G. et Johnson M. (1999). Philosophy in the Flesh : The Embodied Mind and its Challenge to Western Thought. New York : Basic Books.

Langacker R. (1982). "Space Grammar, Analysability, and the English Passive”. Language, vol. 58, No. 1 (March), 22-80.

Langacker R. (1987). Foundations of Cognitive Grammar. Volume 1. Theoretical Prerequisites. Stanford : Stanford University Press.

Langacker, R. (1991a). Foundations of Cognitive Grammar. Volume 2. Descriptive Applications. Stanford : Stanford University Press.

Langacker Ronald. (1991b). Concept, Image, and Symbol. The Cognitive Basis of Grammar. Berlin / New York : Mouton de Gruyter. 
Langacker R. (2000). Grammar and conceptualization. Berlin / New York : Mouton de Gruyter.

Langacker R. (2008). Cognitive Grammar. A Basic Introduction. Oxford : Oxford University Press.

Langacker R. (2010). "Reflections on the Functional Characterization of Spatial Prepositions." CORELA (Cognition, Représentation, Langage) - Numéros thématiques / Espace, Préposition, Cognition.

Lapaire J.-R. (2011). “Grammar, gesture and cognition : insights from multimodal utterances and applications for gesture analysis". Visnyk of Lviv University. Philology Series. Issue 52, 88-103.

Leech G. et Svartvik J. (1994). A Communicative Grammar of English. London : Longman.

Levinson S.C. (2004). Space in Language and Cognition. Explorations in Cognitive Diversity. Cambridge : Cambridge University Press.

McNeill D. (1992). Hand and Mind. What Gestures Reveal about Thought. Chicago : The University of Chicago Press.

McNeill D. (2005). Gesture and Thought. Chicago / London : The University of Chicago Press.

Radden G. et Dirven R. (2007). Cognitive English Grammar. Amsterdam : John Benjamins.

Svorou S. (1994). The Grammar of Space. Amsterdam / Philadelphia : John Benjamins.

Talmy L. (2000). Toward a Cognitive Semantics. Cambridge, Mass. : The MIT Press.

Tommasello M. (2009). Constructing a language. A usage-based model of language-acquisition.

Cambridge, Mass. : Harvard University Press.

Vandeloise C. (1984). “Description of Space in French.” Doctoral dissertation. San Diego :

University of California.

Vandeloise C. (1986). L'espace en français. Paris : Editions du Seuil.

Vandeloise C. (1987). « La préposition à et le principe d'anticipation ». Langue française, 76, 77-111.

Vandeloise C. (2006). “Are there spatial prepositions ?”, in M. Hickmann, S. Robert (éds.), Space in languages : linguistic systems and cognitive categories, John Benjamins, 139-154.

Vandeloise C. (2007). « Le verbe aller : l'affranchissement du contexte d'énonciation immédiat ». Journal of French Language Studies, 17 :3, 343-359.

Victorri B. (2010). « Le localisme à l'épreuve du verbe aller. » CORELA - (Cognition, Représentation, Langage) Espace, Préposition, Cognition. Numéro thématique.

Vandeloise C. (2005). Perspectives spatiales et relativité linguistique, in A.M. Russo, S. Harel (éds.), Lieux propices : l'énonciation des lieux, le lieu de l'énonciation dans les contextes francophones interculturels. Québec : Presses de l'Université Laval, 295-310.

\section{NOTES}

1. Au moment même où Langacker (1987) sort le premier volume de ses Foundations of Cognitive Grammar, Lakoff (1987: 283) inscrit la «spatialisation " au cœur de la sémiologie et de la cognition langagières : "(...) spatial structure is mapped into conceptual structure." (283). Plus loin, il ajoute: "syntactic form is characterized in image-schematic terms-using part-whole schemas, linear order, closeness, centrality, size, containers, etc." (487).

2. "I argue that a clear demarcation between lexicon and grammar is far from evident" (Langacker, $2008:$ 18). 
3. Le corpus (Atwood, 2000) est constitué du premier chapitre du roman The Blind Assassin (2000) intitulé The Bridge.

4. Fortis (2010) montre comment le modèle langackérien (1965-1982) s'élabore à partir de concepts inspirés de la sémantique générative puis adopte une perspective «localiste et gestaltiste ", centrée sur le signe et les constructions. L'influence de Fillmore, Lakoff et Talmy est rappelée.

5. Le fonctionnaliste Givòn parle de "grammar about grammar" (1993 : ix) pour dénoncer les approches formelles de la grammaire. Langacker est moins ironique mais plus précis dans sa condamnation de conceptions qu'il estime déshumanisées et inutilement abstraites de la grammaire : "a system of arbitrary forms based on abstract principles unrelated to other aspects of cognition or human endeavor" (2008:3).

6. "Even the most 'grammatical' of grammatical markers- forms like be, do, of, the infinitival to, agreement markers, case inflections, and derivational affixes- are viewed in CG as meaningful." (Langacker $2008: 2$ ).

7. Les collocations suivantes apparaissent dans le COCA : plunge(d) off (a) (vertical) cliff(s)... a pier... a (picturesque) bridge (over the Hudson)... the road... the pavement... the corniche... the (highest) ridge... the tower (of his castle)... the top of the cataract... On rencontre même her car had plunged off a bridge and into an icy river très proche du texte de Margaret Atwood.

8. Pour sailed off, le COCA fait apparaitre les collocations suivantes : sailed off a / the cliff... the bridge rail... the roof... the road... the coast... the black wall... my face...

9. Le COCA révèle que stepping off se construit peu avec le déterminant zéro (stepping off stage... school), davantage avec $\mathrm{A}(\mathrm{N})$ (stepping off a cliff... a gangway... a curb... a stair... a sandbank...) et très majoritairement avec THE (stepping off the ship... the elevator... the train... the plain... the schoolbus... the precipice... the ladder... the elevator).

10. La consultation du COCA révèle que blow(ing) ou blew around portent soit sur le corps tout entier (métonymiquement évoqué au moyen d'un pronom personnel) soit sur certaines parties privilégiées du corps : blowing around me... her...him... them... her face... his leg... Lorsque l'élément pivot est un objet, il s'agit le plus souvent de porch et de hill.

11. Le COCA fournit par ordre de fréquence descendante : in the broad... bright... dusty... dying... fading... weak... waning... receding... muted... morning... full... ebbing... clear... cheeriest... daylight.

12. "Meaning is equated with conceptualization. Linguistic semantics must therefore attempt (...) the explicit description of abstract entities like thoughts and concepts. The term conceptualization is interpreted quite broadly : it encompasses novel conceptions as well as fixed concepts ; sensory, kinesthetic, and emotive experience ; recognition of the immediate context (social, physical, and linguistic)." (Langacker, 1991b : 2).

13. "(G)rammar reduces to schematic symbolic assemblies" (Langacker, $2008: 22$ ).

14. "For one thing, we engage the world not just physically but also mentally, interacting with other entities through perception and thought. It is quite common for such occurrences to be expressed in the same manner as physical interactions." (Langacker, $2010: 29$ )

15. "[T]he main driving force that can be held responsible for the why grammar is structured the way it is" (Heine, $1997: 14$ ).

16. Pour mémoire, citons la définition de la grammaticalisation de Hopper et Trauggot (2003:1) : "As a termuil referring to a research framework, 'grammaticalization' referts to that part of the study of language change that is concerned with such questions as how lexical items and constructions come into certain linguistic contexts to serve grammatical functions or how grammatical items develop new grammatical functions."

17. "Language structure can best be understood with reference to the conceptual foundations on which it rests." (Heine, 1997 : 16).

18. "[H]uman beings, irrespective of whether they live in Siberia or the Kalahari Desert, have the same intellectual, perceptual and physical equipment; are exposed to the same general kinds of 
experiences; and have the same communicative needs (...) [Yet] there are considerable differences across cultures in the way the environment is conceptualized and communication achieved." (1997:11).

19. Exemples tirés du corpus (Atwood, 2000) : "A hot wind was blowing around my head" ; "I was cold all over" (énonciatrice).

20. Dans le corpus (Atwood, 2000), on note qu'à partir d'un premier repérage par rapport au pont "my sister Laura drove a car off a bridge", des repérages secondaires sont opérés et des axes d'orientation tracés : "she went right through the danger sign" ; "The car fell a hundred feet into the ravine, smashing through the treetops". Les constructions prépositionnelles fonctionnent ici en lien étroit avec des verbes de mouvement: drive, go, fall, smash.

21. On peut considérer que dans "the car sailed off the bridge, then hung suspended in the sunlight, glinting like a dragonfly" (Atwood, 2000), le repère constitué par la lumière solaire est de type absolu dans la nomenclature de Heine (1997 : 12):"[a reference point] independent of the position assumed by the speaker, the hearer, or a particular object."

22. O.E.D. : "Aphetic for aback adv. Old English on baec = into or in the rear. Not found before 14th cent. ; formerly with comparative backer, occas. backermore."

23. O.E.D. : "Old French and French front, < Latin front-em, frōns the forehead."

24. Lié à dun (hill). O.E.D. : "In late Old English dúne, dún, aphetic form of adúne adown adv. and prep. , weakened from old English of dúne off the hill or height."

25. "Conceptualization is anthropocentric: Whenever possible, we use human categories to describe and understand non human ones. Accordingly, the human body provides the most important model for expressing concepts of spatial orientation" (Heine, $1997: 40$ )

26. "Typically, there is a close conceptual association between a given body-part and the corresponding spatial concept. Such an association exists, for example, between the body-part 'back' and the spatial concept 'back', between 'face' and 'front', or between 'head' and 'up' since in all these cases the former item is the most probable diachronic source for the latter." (Heine, $1997: 45)$

27. L'anglais et le français font aussi référence au FRONT (in front of) et à la FACE (facing, « en face de »).

28. "We have to be aware that essentially the only way language is accessible to the analyst is in the form of products resulting from acts of communication. Linguistic communication takes place under specific conditions. These conditions concern the role relationship typically involved in speaker-hearer interaction."(Heine, $1997: 15)$

29. Ordre décroissant des collocations lost in + SN, corpus COCA (450 millions de mots).

30. "Adult linguistic competence is based, to a much larger degree than previously supposed, on concrete pieces of language and straightforward generalizations across them." (Tommasello, $2009: 6)$.

31. La zone active est la partie, facette, etc. de l'entité qui est impliquée au premier plan dans une interaction. Voir Langacker (1991b : 189-201).

32. Dans ces domaines plus abstraits, notent-ils, les constructions prépositionnelles fonctionnent souvent comme des équivalents nominaux de propositions subordonnées (Radden \& Dirven, 2007 : 327) : He cried for help ( = in order to get help). 


\section{RÉSUMÉS}

La grammaire cognitive a commencé par s'appeler « grammaire de l'espace » et ce n'est donc pas un hasard si la cognition spatiale a joué un rôle majeur dans le développement de la théorie générale. De nos jours encore, des termes dénotant des entités spatiales comme domaine, région, espace, scène, zone, cadre, ancrage, repère sont appliqués à un très grand nombre de phénomènes.

Les prépositions sont fondamentalement considérées par les grammairiens cognitivistes comme des marqueurs spatiaux dont le sens premier a été étendu à d'autres domaines. À ce titre, les prépositions révèlent de façon remarquable les mécanismes de représentation qui sous-tendent la représentation symbolique des relations spatiales. Elles sont également des indices fiables des phénomènes de transfert conceptuel entre domaines. Il est d'usage d'attribuer à chaque préposition une forme schématique abstraite, figurée au moyen d'un diagramme. Ce processus spontané de représentation permet de faire comprendre le caractère signifiant de la morphologie grammaticale, de mettre en évidence les figures et les images mentales associées au sens grammatical, de faire ressortir l'écologie et l'ancrage expérientiel des mécanismes de conceptualisation humains, de révéler l'articulation entre motivations notionnelles et fonctionnelles dans le processus de grammaticalisation.

Cognitive Grammar first began as « space grammar » so it is no coincidence that spatial cognition should have played a major role in the development of the general theoretical framework. Terms referring to spatial concepts such as domain, region, space, stage, zone, frame, ground, landmark are still used extensively to describe a wide range of linguistic phenomena, extending beyond spatial organization.

Prepositions are primarily viewed as spatial grams whose basic schematic meanings have been extended to other domains. As such, prepositions reveal the construal operations that underlie the semiotic expression of spatial configurations. They are also sure indicators of conceptual transfer processes. Prepositions are typically ascribed an abstract configurational structure that is usually represented in diagrammatic form. Precious insight is thus provided into the contentfulness and meaningfulness of grammatical morphemes, the patterned nature and imagistic quality of grammatical meaning, the ecology and experiential grounding of human conceptualization strategies, the interplay between notional and functional motivations in grammaticalization processes.

\section{INDEX}

Mots-clés : préposition, marqueur spatial, cognition spatiale, schématisme, opérations de représentation

Keywords : preposition, spatial gram, spatial cognition, schematicity, construal operations 


\section{AUTEUR}

\section{JEAN-RÉMI LAPAIRE}

Université Bordeaux Montaigne

EA CLIMAS

jrlapaire@u-bordeaux-montaigne.fr 